\title{
Identification of putative pathogenic SNPs implied in schizophrenia-associated miRNAs
}

\author{
Xiaohan Sun ${ }^{1,2}$ and Junying Zhang ${ }^{1 *}$
}

\begin{abstract}
Background: Schizophrenia is a severe brain disorder, and SNPs (Single nucleotide polymorphism) in schizophrenia-associated miRNAs are believed to be one of the important reasons for dysregulation which might contribute to the altered expression of genes and ultimately result in the disease. Identification of causal SNPs in associated miRNAs may have certain significance in understanding the mechanism of schizophrenia.

Results: For the above purposes, a method based on detection of free energy change is proposed for identification of causal SNPs in schizophrenia-associated miRNAs. A miRNA is firstly segmented, and free energy change is computed after adding an SNP into a segment. The method discovers successfully 6 out of 32 known SNPs and some artificial SNPs could cause significant change in free energy, and among which, 6 known SNPs are supposed to be responsible for most cases of schizophrenia in population.

Conclusions: The proposed method is not only a convenient way to discover causal SNPs in schizophrenia-associated miRNAs without any biochemical assay or sample comparison between cases and controls, but it also has high resolution for causal SNPs even if the SNPs are not reported for their very rare cases in the population. Moreover, the method can be applied to discover the causal SNPs in miRNAs associated with other diseases.
\end{abstract}

\section{Background}

Single nucleotide polymorphism (SNP) is a single nucleotide variation that occurs when a single nucleotide, such as an Adenine (A), replaces one of the other three nucleotide letters: Thymine $(\mathrm{T})$, Cytosine $(\mathrm{C})$ or Guanine $(\mathrm{G})$, and it is an important variation for the diversity among individuals, as well as leading to phenotypes, traits, and diseases [1]. SNPs usually occur in regions where natural selection is acting and fixating the allele of the SNP that constitutes the most favorable genetic adaptation [2], and most SNPs (93\%) discovered by genome-wide association studies (GWAS) over the last decade that appear to contribute to human disease risk are not located in proteincoding regions [3-5], suggesting that SNPs regulate gene transcription levels rather than alter the protein-coding sequence or protein structure [4]. Therefore, SNPs located in non-coding regions involved in regulation might be closely associated with disease.

\footnotetext{
* Correspondence: jyzhang@mail.xidian.edu.cn

'School of Computer Science and Technology, Xidian University, Xi'an 710071, P. R. China
}

Full list of author information is available at the end of the article
MiRNAs (microRNA) are small 20-24 nucleotide (nt) non-coding RNAs that normally negatively regulate messenger RNAs (mRNAs) translation either via mRNA degradation or repression of mRNA translation [5-7]. Animal genomes harbour numerous small, non-coding miRNAs which post-transcriptionally regulate many protein-coding genes to influence the processes ranging from metabolism, development and regulation of nervous and immune systems to the onset of cancer [8]. To date, hundreds of miRNAs have been identified in the human genome, and they play key roles in a broad range of physiologic and pathologic processes [9]. Indeed, the growing understanding of the regulatory properties and pleiotropic effects of miRNAs on molecular and cellular mechanisms, suggests that alterations in the miRNA/mRNA interaction may contribute to phenotypic variation [10]. SNPs in the miRNAs might affect the expression of multiple target genes by disturbing translation or cleavage of the target mRNAs [11], and exhibit more profound and broader biological effects than SNPs in mRNAs [12]. SNPs in miRNAs affect gene regulation mainly in two ways: either impairing miRNA/ mRNA interaction or disturbing miRNA biogenesis, both of which finally result in gene dysregulation. 
MiRNA/mRNA interaction can be impaired by SNPs in mature miRNAs. SNPs in mature mRNAs could create, destroy, or modify the efficiency of miRNA in binding to 3 '-untranslated region (UTR) of target mRNAs [13-15], hence they might cause post-transcriptional dysregulation due to the stringent recognition requirement in binding mature miRNAs to 3'UTRs of target mRNAs in a sequence-specific manner [16,17].

MiRNA biogenesis can be blocked by SNPs in terminal loops and extension duplexes (covering 25 nt upstream and downstream from the cleavage sites of Drosha in miRNAs) [18]. Maturation of canonical miRNAs is a two-step cleavage of primary miRNA (pri-miRNA) by Drosha and Dicer [19], following which, pri-miRNA is processed into $\sim 22 \mathrm{nt}$ double-stranded RNA product (Figure 1). Successful cleavage can produce miRNA for miRNA/mRNA interaction, but some SNPs might inhibit cleavage, thus block miRNA biogenesis including miRNA processing, strand loading, and so forth $[1,12]$. In the process of miRNA maturation, compared to cleavage by Dicer, cleavage by Drosha is more important because Dicer just cleaves double-stranded RNA (dsRNA) at the site of 22 nt from the cleavage sites of Drosha [20]. The big terminal loop and the stable double-strand of an RNA hairpin are two main requirements for Drosha to effectively cleave pri-miRNA, and an SNP which destroys the two requirements is supposed to block miRNA biogenesis.

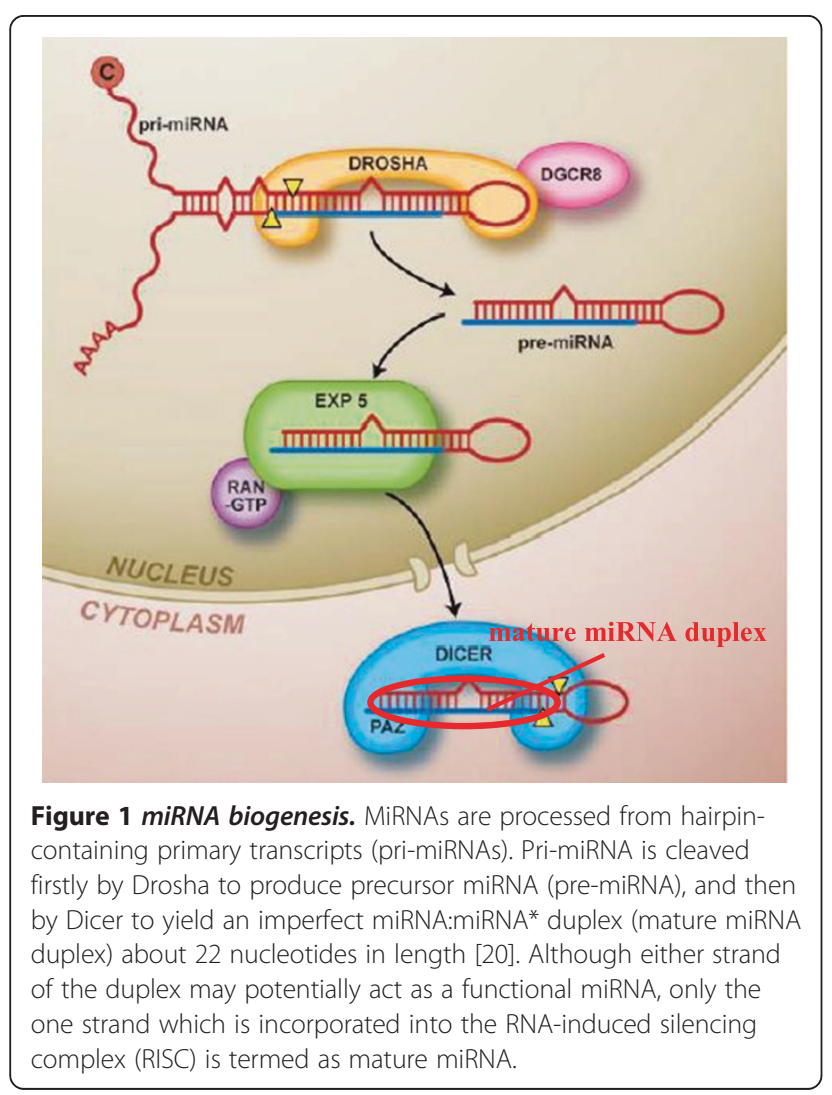

Case-control study is a main route for searching diseaserelated SNPs. By case-control study, some SNPs in genes associated with schizophrenia are revealed [21], some SNPs in pre-miRNA (miRNA precursor) flanking regions are discovered to be associated with schizophrenia or breast cancer $[6,10]$, and some SNPs in pre-miRNAs or in miRNA seeds are detected to cause coronary artery disease [9]. In contrast to traditional case-control studies which specifically test one or a few genetic regions, GWAS investigates the entire genome. For example, Jing Gong et al. performed a genome-wide scan for SNPs in human pre-miRNAs, miRNA flanking regions, and target mRNAs, and designed a pipeline to predict the effects of them on miRNA/mRNA interaction [22]; Keren Oved et al. identified CHL1 as a tentative selective serotonin reuptake inhibitors by genomewide expression profiling of human lymphoblastoid cell lines (LCLs) [23]; and Jesse D. Ziebarth et al. constructed a database, PolymiRTS Database 2.0, which provided links between SNPs in miRNA target sites and the results of GWAS of human diseases [24]. The studies above have discovered disease-related SNPs in miRNAs, but the complicated steps including sample collection, biochemical assay and long-period observation become a limit of convenient and fast detection of disease-related SNPs in miRNAs. Moreover, the studies generally detect the SNPs whose rare frequency is more than $1 \%$, so the rarer SNPs cannot be discovered.

Among a multitude of complex diseases, schizophrenia might be closely associated with miRNA mutation. Different from many complex diseases with obvious changed expression of a few genes, schizophrenia follows many genes with small expression alteration [10]. Because no core genes with significant expression alternation contribute to a big risk of schizophrenia, we might hypothesize that dysregulation of genes could play a more important role in schizophrenia than altered expression genes themselves [10]. As an important regulator of genes, a single miRNA can regulate expression of multiple genes, an SNP in schizophrenia-associated miRNAs (SZmiRNA) can therefore change the expression of multiple genes even if the expression change of each gene is not significant. According to it, we believe that an SNP in SZmiRNAs has a high possibility to cause schizophrenia. Moreover, schizophrenia is a severe disabling brain disease, and miRNAs have been proved to be involved in brain development and function [10,25], therefore, SNPs in SZmiRNAs, a main mutation of miRNAs, are also supposed to be closely associated with schizophrenia. However, the association of SNPs in miRNAs and schizophrenia is unexplored due to poor understanding of the pathophysiology and molecular mechanisms of schizophrenia even if there have been some recent researches focusing on the identification and analysis of SNPs in miRNAs [15,22,26-28]. 
Identification of schizophrenia-causal SNPs (SZ-SNP) is necessary for further study of schizophrenia. In this study, we propose a novel and simple computational method to detect SZ-SNPs in SZmiRNAs by detecting free energy change caused by the existence of an SNP. Using the method, the SNPs which can change free energy significantly are discovered, and 6 out of 32 known SNPs in SZmiRNAs are supposed to be responsible for most cases of schizophrenia in population.

\section{Results}

\section{Identified SNPs associated with schizophrenia}

We collect 20 SZmiRNAs, and add an SNP into them by replacing every nucleotide with any one of the other three letters after dividing each SZmiRNA into 3 segments: terminal loop, mature miRNA duplex and extension duplex (see Methods). There are separately 1284, 3585, and 4065 SNPs in the three segments of the SZmiRNAs. By detecting free energy change of each segment, 176 (13.7\%) SNPs in terminal loops can decrease free energy, and 2310 (64.4\%) SNPs in mature miRNA duplexes and 1775 (43.7\%) SNPs in extension duplexes can increase free energy. Although the $4261(176+2310+1775)$ SNPs might be associated with schizophrenia more or less, many of them are not causal ones because some just change free energy very slightly. We sort the SNPs in terminal loops by the free energy change, and then compute (sum of free energy change of top $n(n=1,2, \ldots, 176)$ SNPs/sum of all free energy change) to find the inflection point of free energy change. The top 57 SNPs in terminal loops are supposed to be causal ones. Similarly, the top 533 SNPs in extension duplexes and 888 SNPs in mature miRNAs are identifies as SZ-SNPs. From the SZ-SNPs (see Additional file 1), we find that free energy is significantly decreased when nucleotides $A$ and $U$ are changed and free energy is significantly increased when nucleotides $C$ and $G$ are changed. This phenomenon comes from that there are three hydrogen bonds between a C-G base pair and two bonds between an A-U base pair, therefore, forming a new or destroying an available $\mathrm{C}-\mathrm{G}$ pair can cause larger change in free energy than an A-U pair.

\section{Identification of SNPs responsible for most cases of schizophrenia}

There are two groups of SNPs in our study: known SNPs which are those detected in former studies, and artificial SNPs which are never reported. By analyzing free energy change caused by SNPs (see Table 1), we find there seems no significant difference between the known and artificial SZ-SNPs. The range of free energy increase caused by all the SZ-SNPs in mature miRNAs is from to 1.5 to 4.7 (average free energy increase is 6.0), and the range of free energy increase caused by the known SZ-SNPs is from 1.7 to 3.4 (average free energy increase is 2.5).
The range of free energy increase caused by all the SNPs in extension duplexes is from to 2.7 to 18 (average free energy increase is 6.0), and the range of free energy increase caused by the known SZ-SNPs is from 2.7 to 18 (average free energy increase is 8.4). We perform a $t$-test and prove that free energy change caused by the known SZ-SNPs is not significantly different from that caused by the artificial SZ-SNPs, but a known SZ-SNPs have higher possibility to be causal for most cases of schizophrenia than an artificial one even if free energy change caused by them is same. People could have schizophrenia if they has any SZ-SNP, either known or artificial, but there could be few people if a SZ-SNP has very low minor allele frequency, therefore, the frequency of a SNP has to be taken into account for identifying causal SNPs in most cases of schizophrenia in population. We think artificial SNPs have a lower minor allele frequency than known SNPs because they never been detected.

According to the minor allele frequency of SNPs, we divide them into three categories: known SNPs with known frequency, known SNPs with unknown frequency, and artificial SNPs. We firstly estimate a random frequency in the known frequency range of the known SNPs for the known SNPs with unknown frequency, and estimate a random frequency between 0 and the smallest known frequency of the known SNPs for the artificial SNPs. We then compute (frequency"free energy change) as a score for each SNP, and sort the SNPs by the score. The process of frequency estimation, score computation and SNP sorting is operated 1000 times, the top SNPs accounting for $50 \%$ of sum of scores (sum of negative scores in terminal loops and sum of positive scores in extension duplexes and mature miRNAs) with $\mathrm{p}$-value $<0.05$ are supposed to be SZ-SNPs responsible for most cases. Among the SZ-SNPs, there are 6 SNPs are discovered, and all of them are known (see Table 2).

One SZ-SNP (rs76481776) among six SZ-SNPs responsible for most cases of schizophrenia has been detected to be associated with major depression and schizophrenia in reference [29], and three known SZ-SNPs (rs80041074, rs77586312, and rs75953509) were discovered in patient samples [30].

\section{Discussion}

\section{SZmiRNAs without significant or known SZ-SNPs}

Although all 27 SZmiRNAs are reported to be associated with schizophrenia [10,29-35], SNPs in them do not have same close association with schizophrenia.

First, some SZmiRNAs do not contain significant SZ-SNPs. There are separately 19, 27, and 19 unique SZmiRNAs which contain significant SZ-SNPs in terminal loops, mature miRNA and extension duplexes. For the SZmiRNAs which do not contain significant SZ-SNPs, SNPs in miRNAs might not be the main ways 
Table 1 Free energy change caused by SNPs

\begin{tabular}{|c|c|c|c|c|c|c|c|c|c|}
\hline \multirow[t]{2}{*}{ Segment } & \multicolumn{3}{|c|}{ SNP } & \multicolumn{3}{|c|}{ SNP with changed free energy } & \multicolumn{3}{|c|}{ SZ-SNP } \\
\hline & Number & $\begin{array}{l}\text { Range of free } \\
\text { energy change }\end{array}$ & $\begin{array}{c}\text { Average free } \\
\text { energy change }\end{array}$ & Number & $\begin{array}{c}\text { Range of } \\
\text { free energy } \\
\text { change }\end{array}$ & $\begin{array}{c}\text { Average } \\
\text { free energy } \\
\text { change }\end{array}$ & Number & $\begin{array}{c}\text { Range of } \\
\text { free energy } \\
\text { change }\end{array}$ & $\begin{array}{c}\text { Average } \\
\text { free energy } \\
\text { change }\end{array}$ \\
\hline Terminal loop & 1284 & {$[-6.2,5.5]$} & 0.08 & 176 & {$[-6.2,0)$} & -1.1 & 57 & {$[-6.2,-1.3]$} & -2.5 \\
\hline Mature miRNA & 3585 & {$[4.7,-3.4]$} & 0.56 & 2310 & {$[4.7,0)$} & 1.3 & 888 & {$[4.7,1.5]$} & 2.2 \\
\hline Extension duplex & 4065 & {$[18.2,-10.9]$} & 0.6 & 1775 & {$[18.2,0)$} & 2.6 & 533 & {$[18.2,2.7]$} & 6.0 \\
\hline
\end{tabular}

SNPs with changed free energy are those causing decrease in free energy of terminal loops and those causing increase in free energy of mature miRNAs and extension duplexes.

to be associated with schizophrenia. Some SZmiRNAs contain more significant SZ-SNPs than others, so the SNPs in the miRNAs are more likely to have a stronger association with schizophrenia. Second, there are four SZmiRNAs (hsa-miR-198-5p, hsa-miR-92b-3p, hsa-miR-182, and hsa-let-7 $g$ in Table 2) could be largely responsible for most cases of schizophrenia caused by SNPs in miRNAs.

As for the SZmiRNAs which contain no SZ-SNPs in some segments, they are still associated with schizophrenia. First, SNPs in other regions, such as schizophreniaassociated genes (SZGenes) or $1 \mathrm{k}$ flanking regions of SZmiRNAs, can cause abnormal expression of SZGenes or SZmiRNAs, thus are associated with schizophrenia. Second, other mutation, such as copy number variation $(\mathrm{CNV})$, or DNA methylation, are also responsible for schizophrenia [36,37].

\section{High frequency of known SNPs in SZmiRNAs}

The total length of 27 SZmiRNAs is 3009 bases, and in which there are 35 known SNPs, thus the frequency of known SNPs in the 27 SZmiRNAs is $1.16 \%$ which is much higher than the average estimate that SNPs occur 1 in 1000 base pairs.

Uneven distribution of SNPs in genome might account for the high frequency of SNPs in SZmiRNAs. First, SNPs usually occur more frequently in non-coding regions than in coding regions $[2,22]$ and miRNAs are very important regulators in non-coding regions, so the frequency of SNPs in SZmiRNAs should be higher than in coding regions. Second, compared with genes, miRNAs haven the more profound and broader influence on natural selection which is acting and fixating the allele of the SNP that constitutes the most favorable genetic adaptation [2,22], thus having a higher frequency of SNPs. Moreover, schizophrenia is likely caused by many genes which individually contribute a small risk [25]. A single SNP in SZmiRNA can change expression of many SZGenes by changing the SZmiRNA/SZGene interaction, so SNPs in SZmiRNAs are more possible to cause schizophrenia than SNPs in other regions [25]. High frequency of SNPs in SZmiRNAs can also explain the important role of miRNA in the etiology of schizophrenia.

\section{Uneven distribution of known SNPs in SZmiRNA segments}

The frequencies of the known SNPs in terminal loops, mature miRNA and extension duplexes of SZmiRNAs are 11.68 SNPs/kb (5), $4.18 \mathrm{SNPs} / \mathrm{kb}$ (5) and 16.24 SNPs/kb (22) separately, and frequencies of the known SZ-SNPs responsible for most cases of schizophrenia in the 3 segments are 0 SZ-SNPs/kb (0 SNP), 1.67 SZ-SNPs/kb (2) and 2.95 SZ$\mathrm{SNPs} / \mathrm{kb}$ (4) separately. The frequencies of known SNPs in the 3 segments of SZmiRNAs are listed in Table 3, from which, we find that the frequency of the known SNPs in extension duplexes is higher than that in terminal loops and mature miRNA duplexes. The higher frequency of known SNPs and SZ-SNPs in extension duplexes suggests that SNPs might be prone to occur in extension duplexes and unstable structure of extension duplexes might have profounder influence on miRNA biogenesis than a small terminal loop.

\section{None of known SNPs which can decrease free energy of terminal loops}

None of the five known SNPs in terminal loops can decrease free energy, but we cannot draw the conclusion

Table 2 SZ-SNPs responsible for most cases of schizophrenia

\begin{tabular}{llllll}
\hline miRNA & Strand & Segment & Sequence & SNP ID & Allele \\
\hline hsa-miR-198-5p & - & mature miRNA & GGUCCAGAGGGGAGAUAGG & rs142303836 & G/A \\
hsa-miR-92b-3p & + & mature miRNA & UAUUGCACUCGUCCCGGCCUCC & rs12759620 \\
hsa-miR-182 & - & extension duplex & UGGGGCGAGGACUCAGCCGGCACCC & rs76481776 & G/A \\
hsa-miR-182 & - & extension duplex & UGGGGCGAGGACUCAGCCGGCACCC & rs374455999 & G/A \\
hsa-miR-182 & - & extension duplex & GGGGAGCUGCUUGCCUCCCCCCGUU & rs370756213 & C/U \\
hsa-let-7 g & - & extension duplex & CAGGAACAGCGCGCCAGCUGCCAAG & rs9631505 & G/A \\
\hline
\end{tabular}

SNPs in sequences are highlighted in bold and italic. 
Table 3 known SNP and SZ-SNP frequencies in SZmiRNAs

\begin{tabular}{ccccc}
\hline Region & $\begin{array}{c}\text { number } \\
\text { of SNPs }\end{array}$ & $\begin{array}{c}\text { Mean } \\
\text { frequency } \\
\text { (number/kb) }\end{array}$ & $\begin{array}{c}\text { number of } \\
\text { SZ-SNPs }\end{array}$ & $\begin{array}{c}\text { Mean } \\
\text { frequency } \\
\text { (number/kb) }\end{array}$ \\
\hline Terminal loop & 5 & 11.68 & 0 & 0 \\
Mature miRNA & 5 & 4.18 & 2 & 1.67 \\
Extension duplex & 22 & 16.24 & 4 & 2.95 \\
\hline
\end{tabular}

that the known SNPs in terminal loops of SZmiRNAs are not associated with schizophrenia because the structure of a terminal loop predicated by software is not accurate. Reference [38] indicates that a real miRNA hairpin structure often holds a large $(>=10 \mathrm{nt})$ and unstructured terminal loop which is a good substrate for efficient cleavage by Drosha, but the size of terminal loop predicted by software is usually small $(<=5 \mathrm{nt}$ ). For instance, using RNAfold [39] (a software to predict the secondary structure of a single stranded RNA or DNA sequences), hsa-let-7 g, hsa-mir-7-1 and hsa-mir-9-2 are predicted to have a very small terminal loop with the size of $4 \mathrm{nt}$, and $h s a-m i r-29 c$ is predicted to have a terminal loop with the size of $5 \mathrm{nt}$. Therefore, the proposed method based on analysis of free energy for detecting SZ-SNPs does not perform in terminal loops as well as in mature miRNA and extension duplexes.

\section{Conclusions}

Some SNPs in SZmiRNAs can change the internal energy of miRNAs by changing miRNA secondary structure or miRNA/mRNA interaction, which can cause abnormal expression of SZmiRNAs and SZGenes, we therefore think that there is a causal link between SNPs in SZmiRNAs and schizophrenia. Moreover, it is feasible to discover causal SNPs by investigating free energy change because free energy is an indicator of structural stability. The proposed method based on free energy for identifying causal SNPs in SZmiRNAs is not only convenient because biochemical assay or sample comparison between cases and controls are not necessary, but it also has high resolution for causal SNPs even if the SNPs never been reported because they are very rare in the population. In addition, the proposed method can be applied to discover the causal SNPs in miRNAs associated with other diseases.

\section{Methods}

Hairpin is the typical structure of a miRNA, which is also known as stem-loop structure. In the structure, base pairs form a double helix (stem) that ends in an unpaired loop (loop). The unpaired loop at the end is the terminal loop, and two mature miRNAs originated from opposite arms of a same miRNA can inhibit gene expression by binding them with 3'UTR of mRNAs. The stable hairpin of a miRNA and stable base-pairing between mature miRNA and target mRNA are the basis for successful cleavage of mature miRNA and inhibition of target mRNA expression, but the stable structures could be changed by SNPs in the miRNA. If target mRNAs of a miRNA are associated with some diseases, the variation in structure stability of a miRNA might cause diseases because expression of target mRNAs cannot be inhibited normally.

An SNP in a miRNA is supposed to be a causal or functional one if it blocks miRNA maturation or hampers miRNA/mRNA interaction, which often presents in a structural variation of the miRNA. In the hairpin of a miRNA, a large terminal loop and stable doublestranded structure of extension duplex (25 nt upstream and downstream sequences from cleavage sites of mature miRNA duplex) are important for miRNA maturation because a large (> = $10 \mathrm{nt})$ terminal loop $[38,40]$ and stable double-stranded structure of an extension duplex make a good substrate for efficient cleavage of a miRNA. An SNP in a terminal loop or an extension duplex can block maturation of miRNA, and result in abnormal expression of target genes for no enough mature miRNA for binding target genes if it decreases the size of the terminal loop or impairs stability of the extension duplex. Moreover, an SNP in mature miRNA can cause abnormal expression of target genes if it destroys base pairs between mature miRNA and 3'UTR of target genes. However, a miRNA mutant with changed structure but retained stability still supports cleavage [18] and miRNA/mRNA interaction, therefore, only the SNPs which can impair stability are considered to be causal.

According to the effect of different parts of a miRNA sequence on miRNA biogenesis and miRNA/mRNA interaction, a miRNA is divided into three segments: terminal loop, mature miRNA duplex and extension duplex. Some structure variations of the three segments with changed stability, such as decrease in the size of a terminal loop, damage in stability of an extension duplex, and destruction of base pairs between miRNA and target genes, can be evaluated conveniently with free energy. Free energy is a reflection of structural stability, and it has been widely used in target prediction, function analysis of miRNA and miRNA/mRNA interaction [17,41-43]. In the hairpin of a miRNA, free bases unpaired with any bases are more active than paired bases because they are easier to act with other free bases, thus accounting for structural instability. Generally, the lower free energy a RNA strand possesses, the more stable its structure is. Free energy here can be used to evaluate the stability of the hairpin of a miRNA and miRNA/mRNA interaction. Although structure variation could not accompany a changed free energy, and a structure variation with an unchanged free energy has few effect on stability [18], change in free energy does follow structure variation, and a structure variation with changed free energy must change stability. 
An SNP in a SZmiRNA can be identified as a causal one by detecting free energy change from addition of addition of the SNP.

The proposed method for SZ-SNPs in SZmiRNAs based on free energy detection is composed of three steps: SZmiRNA segmentation, SNP addition, and free energy change detection.

A flowchart of identification procedure is shown in Figure 2.

\section{Collection of SZmiRNAs, SZGenes and SNPs in SZmiRNAs}

Most SZmiRNAs and all SZGenes are downloaded from Schizophrenia Gene Resource (SZGR, http://bioinfo.mc. vanderbilt.edu/SZGR/), hsa-miR-30e and hsa-miR-182 are collected from some studies associated with mental illness [10,29,30,33-35] (see Additional file 2 and Additional file 3). All the SNPs in the SZmiRNAs are gathered from NCBI (the National Center for Biotechnology Information advances science and health by providing access to biomedical and genomic information, http://www.ncbi.nlm.nih.gov) (see Additional file 4).

\section{Segmentation of SZmiRNAs}

Each SZmiRNA is divided into three segments: terminal loop, mature miRNA duplex, and extension duplex. For example, the segmentation of $h s a-m i R-29 c$ is shown in Figure 3. The segmentation sites of a SZmiRNA are determined based on annotations in miRBase (a miRNA database, http://www.mirbase.org), but there are two exceptional cases. First, some SZmiRNAs have only one mature miRNA sequence annotated in miRBase (e.g. has-miR-206, has-miR-30d, and has-miR-7-3). We must artificially annotate another mature miRNA sequence for them. Two general principles are used for the artificial
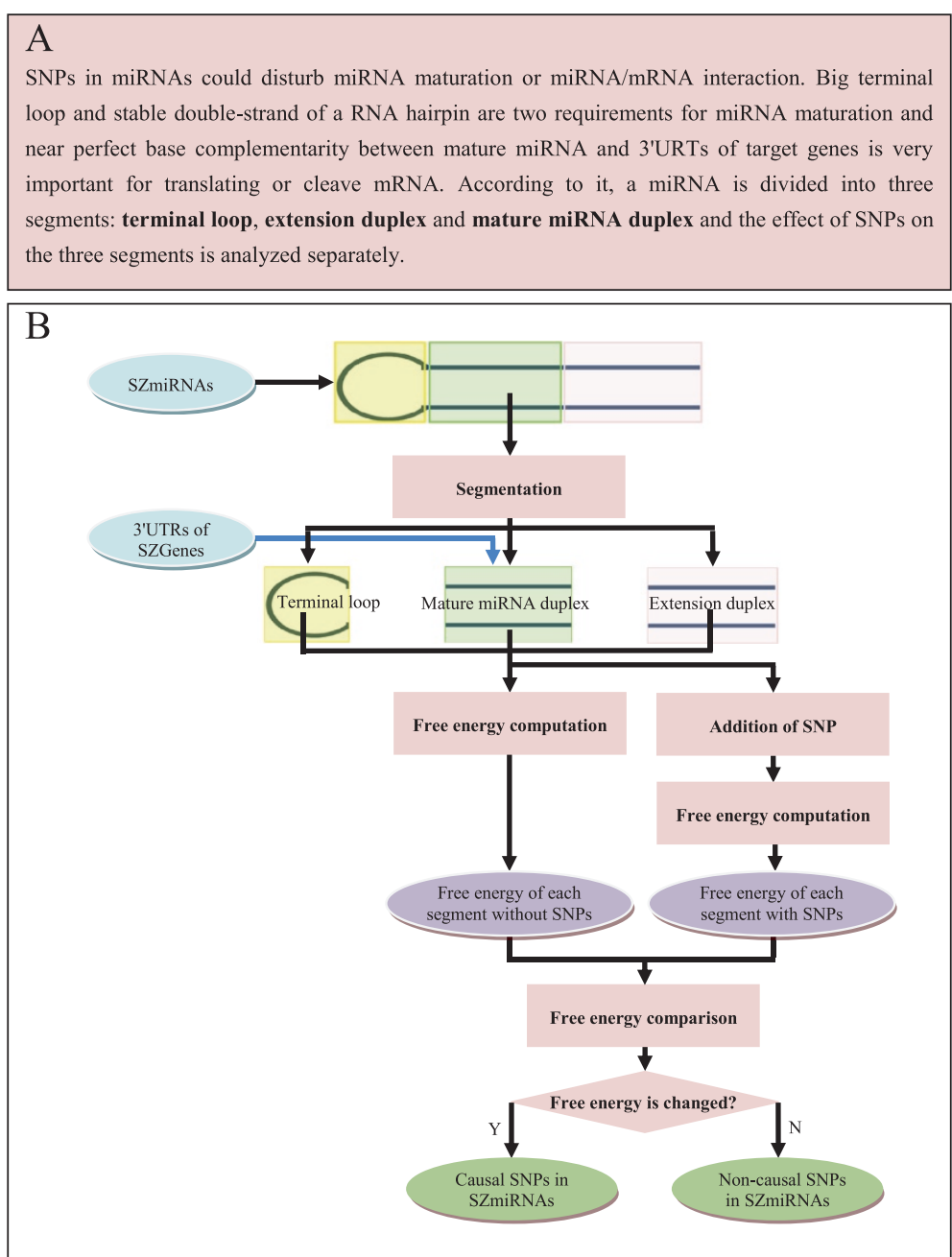

Figure 2 Flowchart of the method. (A) Basis of segmentation, (B) Flowchart to search causal SNPs. Based on the requirements of miRNA maturation and miRNA/mRNA interaction in $\mathbf{A}$, a miRNA is divided into three segments: terminal loop, mature miRNA duplex and extension duplex in B. B presents the procedure to discover causal SNPs in detail. 


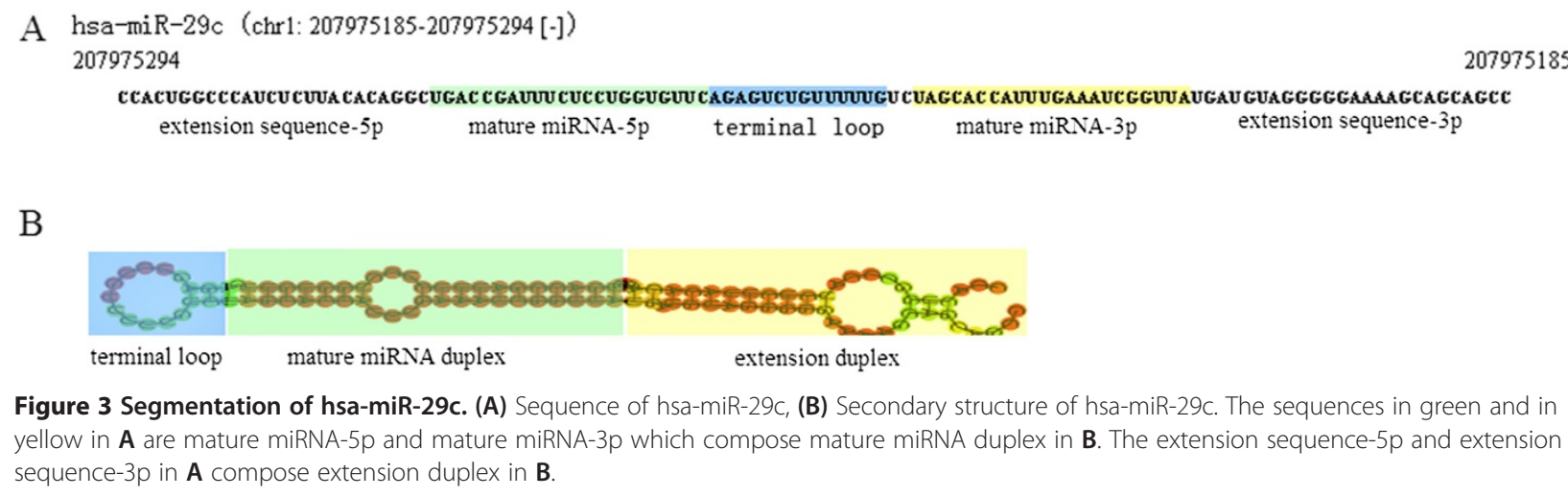

Figure 3 Segmentation of hsa-miR-29c. (A) Sequence of hsa-miR-29c, (B) Secondary structure of hsa-miR-29c. The sequences in green and in yellow in $\mathbf{A}$ are mature miRNA-5p and mature miRNA-3p which compose mature miRNA duplex in $\mathbf{B}$. The extension sequence-5p and extension sequence-3p in $\mathbf{A}$ compose extension duplex in $\mathbf{B}$.

annotation of the mature miRNA sequence: a 2 nt overhang at the start site of mature miRNA-5p sequence and the end site of mature miRNA-3p, and good complementarity between two mature miRNA sequences. Second, there could be overlap between different segments, for instance, hsa-miR-198 has an overlap of three bases between mature miRNA-5p and terminal loop. The overlap of two segments should be contained in both two segments. Segmentation of hsa-miR-198 is shown in Figure 4.

\section{Detection of free energy change from addition of an SNP}

SZ-SNPs are identified by investigating free energy change of each segment caused by the addition of an SNP. After the free energy of each original segment is computed, an SNP is added into each segment by replacing every nucleotide with any one of the other three letters, and the new free energy of the segment with an added SNP is computed. The free energy change caused by the SNP is finally obtained. Below, we analyze the effect of an SNP on the three segments separately.

First, the effect of an SNP on the size of a terminal loop is analyzed. Terminal loop is an unstructured loop in the hairpin of a miRNA, that is, no bases can be paired with others in a terminal loop. An SNP which can decrease the size of a terminal loop is supposed to be a causal one because a large terminal loop is a good substrate for miRNA maturation. If an SNP in a terminal loop makes some bases pair with each other, the size of the terminal loop will be decreased because the complementary bases can form base pairs and they are mistaken for a part of the stem of the hairpin. For example, the sixteen nucleotide variation from $U$ to $G$ in the terminal loop of $h s a-m i R-29 b-2$
(AUUUUUCCAUCUUUGUAU) significantly decreases the size of the terminal loop from 16 to 4 (Figure 5). The decreased size of a terminal loop means there appear fewer free bases, therefore the terminal loop with decreased size certainly follows a decrease in free energy. We firstly compute the free energy of the original terminal loop of a SZmiRNA and that of the terminal loop after adding an SNP, then we can get the free energy change caused by the SNP. The SNPs which can decrease free energy of terminal loops of SZmiRNAs are supposed to be associated with schizophrenia because they can decrease the size of terminal loops and block SZmiRNA maturation. Here, the function rnafold() in Bioinformatics toolbox in Matlab is applied to compute the free energy of terminal loops.

Second, the effect of SNPs in extension duplexes on structural stability can also be evaluated by free energy. Because the stable double-strand of an extension duplex is one of the requirements for miRNA maturation, an SNP in an extension duplex is supposed to be a causal one if it destroys the stability of the extension duplex by destroying base pairs. Destruction of base pairs inevitably releases free bases, and leads to increase in free energy, therefore, the SNPs in extension duplexes which can increase free energy are identified as SZ-SNPs. Here, we also use the function rnafold() to predict free energy of an extension duplex, but the function can only predict free energy of a single strand, and there are two separate sequences in an extension duplex. According to the reference [44], a linker "GGCGGGG" can be inserted between the two sequences of an extension duplex to compose a single strand before computing free energy.

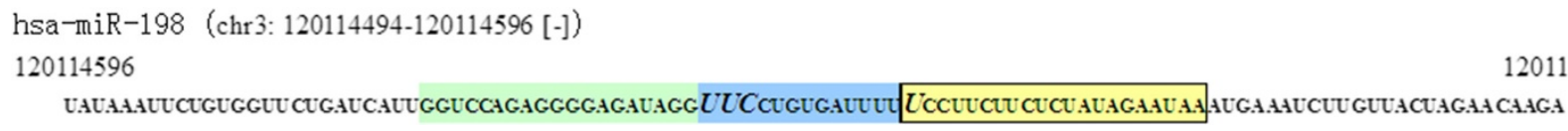

Figure 4 Segments of hsa-miR-198. Mature miRNAs annotated artificially are shown in box and overlapping bases are shown in larger font size and italic. 

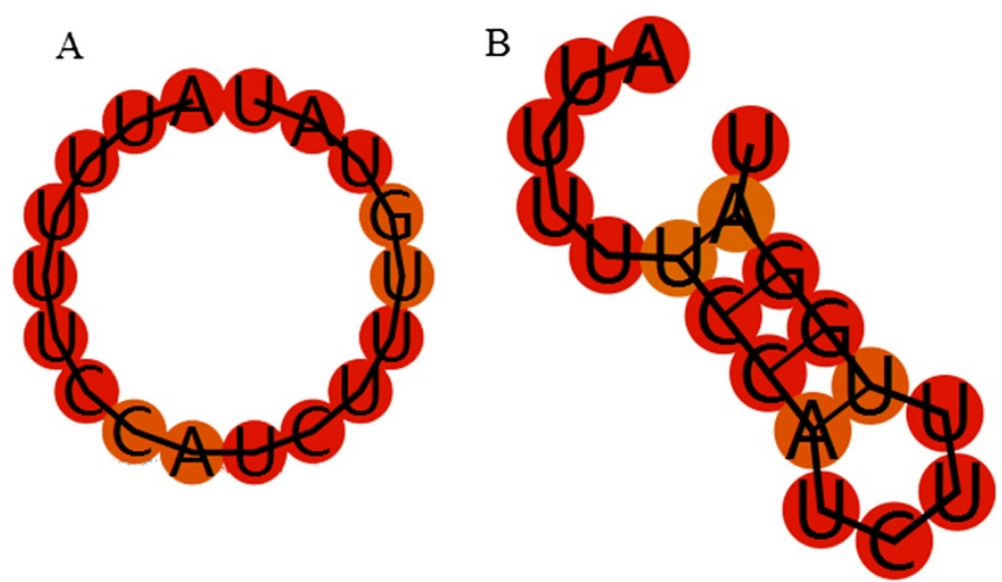

Figure 5 Secondary structure of terminal loop of hsa-miR-29b-2. (A) Secondary structure of original terminal loop of hsa-miR-29b-2, (B) Secondary structure of terminal loop of hsa-miR-29b-2 with the sixteenth base varied from $U$ to $G$. Both the two secondary structures are predicted by RNAfold (software to predict the secondary structures of single stranded RNA or DNA sequences).

Third, the effect of SNPs on miRNA/mRNA interaction is also evaluated by free energy. Mature miRNAs can inhibit protein translation of target mRNAs by binding themselves to 3'UTR of target mRNAs, and good sequence complementarity between miRNAs and target mRNAs is a basic requirement for binding. An SNP in a mature miRNA could also release free bases by destroying base pairs between the mature miRNA and 3'UTRs of its target mRNAs, and cause increase in free energy. Because 3'UTR of a mRNA is a much longer RNA sequence than miRNA and the function rnafold() cannot find the most complementary short sequence in 3'UTR with mature miRNA, RNAhybrid [45] (a on-line tool for finding the minimum free energy hybridization of a long and a short RNA, http://bibiserv.techfak.uni-bielefeld.de/ rnahybrid/submission.html) is applied to compute free energy between a mature miRNA and its target mRNAs.

The detection of a SZ-SNP in a mature SZmiRNA duplex follows the three steps. Step 1, the software Targetscan [46] (http://www.targetscan.org/vert_61, a software to search for predicted miRNA targets in mammals) is used to predict all target genes of a SZmiRNA, from which, the genes also appear in SZGenes are selected as target SZGenes (see Additional file 5). Step 2, all 3'UTRs of the SZGenes are downloaded from UTRdb (a curated database of $5^{\prime}$ and $3^{\prime}$ untranslated sequences of eukaryotic mRNAs, http://utrdb.ba.itb.cnr.it/) and free energy between mature SZmiRNAs and 3'UTRs of target SZGenes is computed by using RNAhybrid. Moreover, one gene might have multiple different 3'UTRs due to multiple transcripts of a gene, but only the 3'UTR with the lowest free energy between which and mature miRNA is taken into account in next step due to the high similarity of 3'UTRs among different transcripts of a gene. Step 3 , the average free energy between a mature SZmiRNA and its all target SZGenes is computed, and the SNPs in mature SZmiRNAs which can increase average free energy are supposed to be associated with schizophrenia.

\section{Additional files}

Additional file 1: Identified SZ-SNPs. There are identified SZ-SNPS in terminal loops, mature miRNAs and extension duplexes of SZmiRNAs.

Additional file 2: SZmiRNAs. SZmiRNAs are collected from 8 studies associated with mental illnesses.

Additional file 3: SZGenes. SZGenes are collected from 4 literatures. Additional file 4: SNPs in SZmiRNAs. SNPs in SZmiRNAs are collected from NCBI.

Additional file 5: Regulatory relation between SZmiRNAs and SZGenes. Target SZGenes are the genes both predicted by the software Targetscan and appeared in SZGenes.

Competing interests

The authors declare that they have no competing interests.

Authors' contributions

XS designed the project, collected and analyzed the data and wrote the manuscript. JZ supervised the study and revised the manuscript. Both authors critically reviewed and approved the final manuscript.

\section{Acknowledgements}

This work was supported by the Natural Science Foundation of China under Grants 61070137, 91130006, 61201312, Research Fund for the Doctoral Program of Higher Education of China (No. 20130203110017), the Fundamental Research Funds for the Central Universities of China BDY171416 and JB140306, the Subject Fund for Shaanxi Province of China 14SXZD014, and Technology Innovation Fund for Weinan of China 2013JCYJ-10.

In addition, Yuanyuan Zhang and Aimin Li revised the manuscript.

\section{Author details}

'School of Computer Science and Technology, Xidian University, Xi'an 710071 , P. R. China. ${ }^{2}$ College of Mathematics and Information Science, Weinan Normal University, Weinan 714099, P. R. China.

Received: 5 January 2014 Accepted: 11 June 2014 Published: 17 June 2014 


\section{References}

1. Bruno AE, Li L, Kalabus JL, Pan Y, Yu A, Hu Z: miRdSNP: a database of disease-associated SNPs and microRNA target sites on 3'UTRs of human genes. BMC Genomics 2012, 13:44.

2. Barreiro LB, Laval G, Quach H, Patin E, Quintana-Murci L: Natural selection has driven population differentiation in modern humans. Nat Genet 2008 40:340-345

3. Coetzee SG, Rhie SK, Berman BP, Coetzee GA, Noushmehr H: FunciSNP: an $\mathrm{R} /$ bioconductor tool integrating functional non-coding data sets with genetic association studies to identify candidate regulatory SNPs. Nucleic Acids Res 2012, 40:e139.

4. Kumar V, Westra HJ, Karjalainen J, Zhernakova DV, Esko T, Hrdlickova B, Almeida R, Zhernakova A, Reinmaa E, Vosa U, Hofker MH, Fehrmann RS, Fu J. Withoff S, Metspalu A, Franke L, Wijmenga C: Human disease-associated genetic variation impacts large intergenic non-coding RNA expression. PLoS Genet 2013, 9:e1003201

5. Richardson K, Lai CQ, Parnell LD, Lee YC, Ordovas JM: A genome-wide survey for SNPs altering microRNA seed sites identifies functional candidates in GWAS. BMC Genomics 2011, 12:504

6. Bensen JT, Tse CK, Nyante SJ, Barnholtz-Sloan JS, Cole SR, Millikan RC: Association of germline microRNA SNPs in pre-miRNA flanking region and breast cancer risk and survival: the Carolina breast cancer study. Cancer Causes Control 2013, 24:1099-1109.

7. Lu M, Shi B, Wang J, Cao Q, Cui Q: TAM: a method for enrichment and depletion analysis of a microRNA category in a list of microRNAs. BMC Bioinformatics 2010, 11:419.

8. Friedlander MR, Chen W, Adamidi C, Maaskola J, Einspanier R, Knespel S, Rajewsky N: Discovering microRNAs from deep sequencing data using miRDeep. Nat Biotechnol 2008, 26:407-415.

9. Zhi H, Wang L, Ma G, Ye X, Yu X, Zhu Y, Zhang Y, Zhang J, Wang B: Polymorphisms of miRNAs genes are associated with the risk and prognosis of coronary artery disease. Clin Res Cardiol 2012, 101:289-296.

10. Hansen $T$, Olsen $L$, Lindow M, Jakobsen KD, Ullum H, Jonsson $E$, Andreassen OA, Djurovic S, Melle I, Agartz I, Hall H, Timm S, Wang AG, Werge T: Brain expressed microRNAs implicated in schizophrenia etiology. PLoS One 2007, 2:e873.

11. Nam JW, Shin KR, Han J, Lee Y, Kim VN, Zhang BT: Human microRNA prediction through a probabilistic co-learning model of sequence and structure. Nucleic Acids Res 2005, 33:3570-3581.

12. Sun G, Yan J, Noltner K, Feng J, Li H, Sarkis DA, Sommer SS, Rossi JJ: SNPs in human miRNA genes affect biogenesis and function. RNA 2009, 15:1640-1651.

13. Zou M, Li D, Lv R, Zhou Y, Wang T, Liu J, Tao C, Ying B, Wang L: Association between two single nucleotide polymorphisms at corresponding microRNA and schizophrenia in a Chinese population. Mol Biol Rep 2012, 39:3385-3391.

14. Amos W: Even small SNP clusters are non-randomly distributed: is this evidence of mutational non-independence? Proc Biol Sci 2010, 277:1443-1449.

15. Saunders MA, Liang H, Li WH: Human polymorphism at microRNAs and microRNA target sites. Proc Natl Acad Sci U S A 2007, 104:3300-3305.

16. Davis BN, Hilyard AC, Nguyen PH, Lagna G, Hata A: Smad proteins bind a conserved RNA sequence to promote microRNA maturation by Drosha. Mol Cell 2010, 39:373-384.

17. Haas U, Sczakiel G, Laufer SD: MicroRNA-mediated regulation of gene expression is affected by disease-associated SNPs within the 3'-UTR via altered RNA structure. RNA Biol 2012, 9:924-937.

18. Lee Y, Ahn C, Han J, Choi H, Kim J, Yim J, Lee J, Provost P, Radmark O, Kim $\mathrm{S}$, Kim VN: The nuclear RNase III Drosha initiates microRNA processing. Nature 2003, 425:415-419.

19. Auyeung VC, Ulitsky I, McGeary SE, Bartel DP: Beyond secondary structure: primary-sequence determinants license pri-miRNA hairpins for processing. Cell 2013, 152:844-858.

20. Cullen BR: Transcription and processing of human microRNA precursors Mol Cell 2004, 16:861-865.

21. Zhou Y, Wang J, Lu X, Song X, Ye Y, Zhou J, Ying B, Wang L: Evaluation of six SNPs of MicroRNA machinery genes and risk of schizophrenia. J Mol Neurosci 2013, 49:594-599.

22. Gong J, Tong Y, Zhang HM, Wang K, Hu T, Shan G, Sun J, Guo AY: Genome-wide identification of SNPs in microRNA genes and the SNP effects on microRNA target binding and biogenesis. Hum Mutat 2012, 33:254-263.
23. Oved K, Morag A, Pasmanik-Chor M, Oron-Karni V, Shomron N, Rehavi M, Stingl JC, Gurwitz D: Genome-wide miRNA expression profiling of human lymphoblastoid cell lines identifies tentative SSRI antidepressant response biomarkers. Pharmacogenomics 2012, 13:1129-1139.

24. Ziebarth JD, Bhattacharya A, Chen A, Cui Y: PolymiRTS Database 2.0: linking polymorphisms in microRNA target sites with human diseases and complex traits. Nucleic Acids Res 2012, 40:D216-D221.

25. Guo AY, Sun J, Jia P, Zhao Z: A novel microRNA and transcription factor mediated regulatory network in schizophrenia. BMC Syst Biol 2010, 4:10.

26. Bhartiya D, Laddha SV, Mukhopadhyay A, Scaria V: miRvar: a comprehensive database for genomic variations in microRNAs. Hum Mutat 2011, 32:E2226-E2245

27. Zorc M, Skok DJ, Godnic I, Calin GA, Horvat S, Jiang Z, Dovc P, Kunej T: Catalog of microRNA seed polymorphisms in vertebrates. PLoS One 2012, 7:e30737.

28. Kim WH, Min KT, Jeon YJ, Kwon Cl, Ko KH, Park PW, Hong SP, Rim KS, Kwon SW, Hwang SG, Kim NK: Association study of microRNA polymorphisms with hepatocellular carcinoma in Korean population. Gene 2012, 504:92-97.

29. Kohen R, Dobra A, Tracy JH, Haugen E: Transcriptome profiling of human hippocampus dentate gyrus granule cells in mental illness. Trans/ Psychiatry 2014, 4:e366.

30. Saus E, Soria V, Escaramis G, Vivarelli F, Crespo JM, Kagerbauer B, Menchon JM, Urretavizcaya M, Gratacos M, Estivill X: Genetic variants and abnormal processing of pre-miR-182, a circadian clock modulator, in major depression patients with late insomnia. Hum Mol Genet 2010, 19:4017-4025.

31. Beveridge NJ, Tooney PA, Carroll AP, Gardiner E, Bowden N, Scott RJ, Tran N, Dedova I, Cairns MJ: Dysregulation of miRNA 181b in the temporal cortex in schizophrenia. Hum Mol Genet 2008, 17:1156-1168.

32. Perkins DO, Jeffries CD, Jarskog LF, Thomson JM, Woods K, Newman MA Parker JS, Jin J, Hammond SM: microRNA expression in the prefrontal cortex of individuals with schizophrenia and schizoaffective disorder. Genome Biol 2007, 8:R27

33. Xu Y, Li F, Zhang B, Zhang $K$, Zhang F, Huang $X$, Sun N, Ren Y, Sui M, Liu P: MicroRNAs and target site screening reveals a pre-microRNA-30e variant associated with schizophrenia. Schizophr Res 2010, 119:219-227.

34. Im HI, Kenny PJ: MicroRNAs in neuronal function and dysfunction. Trend Neurosci 2012, 35:325-334

35. Potkin SG, Macciardi F, Guffanti G, Fallon JH, Wang Q, Turner JA, Lakatos A Miles MF, Lander A, Vawter MP, Xie X: Identifying gene regulatory networks in schizophrenia. Neuroimage 2010, 53:839-847.

36. Hosak L, Silhan P, Hosakova J: Genomic copy number variations: a breakthrough in our knowledge on schizophrenia etiology? Neuro Endocrinol Lett 2012, 33:183-190.

37. Liu J, Chen J, Ehrlich S, Walton E, White T, Perrone-Bizzozero N, Bustillo J, Turner JA, Calhoun VD: Methylation patterns in whole blood correlate with symptoms in schizophrenia patients. Schizophr Bull 2013. sbt080.

38. Zeng Y, Yi R, Cullen BR: Recognition and cleavage of primary microRNA precursors by the nuclear processing enzyme Drosha. EMBO J 2005 24:138-148

39. Gruber AR, Lorenz R, Bernhart SH, Neubock R, Hofacker IL: The Vienna RNA websuite. Nucleic Acids Res 2008, 36:W70-W74

40. Zeng $Y$, Cullen BR: Sequence requirements for micro RNA processing and function in human cells. RNA 2003, 9:112-123.

41. Kertesz M, lovino N, Unnerstall U, Gaul U, Segal E: The role of site accessibility in microRNA target recognition. Nat Genet 2007, 39:1278-1284.

42. Long D, Lee R, Williams P, Chan CY, Ambros V, Ding Y: Potent effect of target structure on microRNA function. Nat Struct Mol Biol 2007, 14:287-294.

43. Robins H, Li Y, Padgett RW: Incorporating structure to predict microRNA targets. Proc Natl Acad Sci U S A 2005, 102:4006-4009.

44. Miranda KC, Huynh T, Tay Y, Ang YS, Tam WL, Thomson AM, Lim B, Rigoutsos I: A pattern-based method for the identification of MicroRNA binding sites and their corresponding heteroduplexes. Cell 2006, 126:1203-1217.

45. Kruger J, Rehmsmeier M: RNAhybrid: microRNA target prediction easy, fast and flexible. Nucleic Acids Res 2006, 34:W451-W454.

46. Lewis BP, Shih $\mathrm{H}$, Jones-Rhoades MW, Bartel DP, Burge CB: Prediction of mammalian microRNA targets. Cell 2003, 115:787-798.

doi:10.1186/1471-2105-15-194

Cite this article as: Sun and Zhang: Identification of putative pathogenic SNPs implied in schizophrenia-associated miRNAs. BMC Bioinformatics 2014 15:194. 